\title{
C-SPECT: a novel cardiac single-photon emission computed tomography system
}

\author{
J. Strologas ${ }^{* a, c}$, M. Rozler ${ }^{c}$, S. Metzler ${ }^{b}$, D. Stentz ${ }^{b}$, S. Poopalasingam ${ }^{b}$, W. Chang $^{b}$ \\ ${ }^{a}$ University of Ioannina, Ioannina, Greece, \\ ${ }^{b}$ University of Pennsylvania, Philadelphia, USA. \\ ${ }^{c}$ Rush University Medical Center, Chicago, USA.
}

\begin{abstract}
Single-photon emission computed tomography (SPECT) is the leading medical-imaging method for the study myocardial perfusion, which is important for the diagnosis and treatment of coronary-artery disease, the number-one killer in the western world. C-SPECT is a proposed dedicated cardiac SPECT system designed to achieve at least double the geometric efficiency compared to general-purpose dual-head gamma cameras, for the same resolution. This improvement can be used to reduce patient radiation dose, achieve fast or dynamic imaging, and enhance the quality of images. The system consists of stationary detector modules of pixelated $\mathrm{NaI}(\mathrm{Tl})$, a slit-slat collimator with interchangeable slits and collapsible slats, and an integrated CT for attenuation correction. The collimator slits provide pinhole collimation in the transverse plane, whereas the slats offer parallel-beam collimation in the axial direction. The adaptive power of the collimator allows us to adjust, in situ, the sensitivity and resolution depending on the imaging task. This way, superior reconstructed-image resolution could be achieved if the system operates with the usual geometric efficiency of the industry's benchmark. The system gantry wraps around patients' left-front thorax and provides a transverse projection minification of $\sim 50 \%$, for a maximal number of minimally-overlapping projections, given the limitations from the spatial resolution of the pixelated detector. We present the design principles and preliminary imaging performance using three-dimensional iterative reconstruction with resolution recovery and data from the newly-built laboratory prototype as well as Monte-Carlo (MC) simulations of the full system.
\end{abstract}

This work was supported by NIH grant HL108119 and utilized resources provided by the Open Science Grid, which is supported by the National Science Foundation and the U.S. Department of Energy's Office of Science.

38th International Conference on High Energy Physics

3-10 August 2016

Chicago, USA

* presenter 


\section{Introduction}

Cardiac single-photon emission computed tomography (SPECT) is based on the acquisition of several two-dimensional projections of three-dimensional distributions of photons emitted from the heart via an injected radiopharmaceutical that follows the blood flow. Three-dimensional reconstruction of the radioactivity distribution in the heart translates to the corresponding radiopharmaceutical uptake. Any areas of the myocardium that demonstrate lower uptake could suffer from lower blood supply due to, for example, coronary-artery disease.

Unlike positron-emission tomography (PET), where the line of response is determined by a pair of back-to-back photons, in SPECT the origin of the detected photon is constrained by the presence of a collimator, which improves the quality but dramatically reduces the amount of detected data: roughly, only one for every $\sim 10^{4}$ generated photons are eventually detected. Higher collimator sensitivity, i.e., wider collimator holes, improves the statistical uncertainty of the detected photon counts but worsens the systematic uncertainty associated with the photons' origins. Increasing the collimator's resolution by reducing the width of collimator holes has the opposite effect. This resolution-versus-sensitivity compromise is a basic problem for every imaging task.

Cardiac SPECT is traditionally performed with gamma cameras typically comprised of two movable heads, each of which containing a scintillating detector and a collimator. Since their invention, these cameras have been successful for imaging several organs (e.g., heart, liver, brain, etc.) and providing excellent angular sampling (i.e., a large number of projections). Nevertheless, there is room for improvement. They are not optimized for cardiac imaging, nor can they perform dynamic imaging. Their resolution of $\sim 10 \mathrm{~mm}$ could be enhanced for better diagnosis, and their geometric efficiency of about $2 \times 10^{-4}$ could also be increased.

\section{The C-SPECT project}

C-SPECT [1] is a proposed compact cardiac SPECT platform that offers adaptive imaging through multiple collimator configurations. It consists of a stationary system of pixelated detectors and a slit-slat collimator with interchanging lead slits and collapsible tungsten slats. This collimator system offers pinhole-like collimation (slits) in the transverse plane, which is ideal for imaging the compact size of the heart, and parallel-beam collimation (slats) in the axial direction, which is ideal for imaging an extended area of the patient's body, including part of the liver, the main source of background. The gantry with the conveyer that holds a number of slits and exchanges them by rotation follows the body-contour of a large patient. To maximize the number of projections, an image minification of about $50 \%$ is used, leading to 16 projections for the standard scanning mode. A pre-determined cylindrical imaging volume (PIV) is defined by the slits' field of view and offers maximal sensitivity compared to rest of body. The heart of the patient, the position of which is determined by an initial scout scan, is moved into the PIV prior to the main SPECT scan. The slats can be collapsed in order to double the parallel-beam openings and offer larger sensitivity. The adaptive nature of collimation allows us to address the sensitivity-versus-resolution compromise on a patient-by-patient or study-by-study manner. The high-sensitivity slit collimator offers at least 2.5 -times the sensitivity for a resolution equal to the industry's gamma-camera benchmark. The higher sensitivity for same resolution can be utilized for faster imaging or lower patient radiation 
(a)

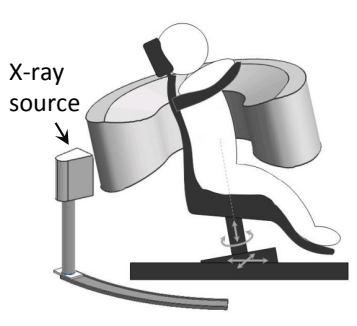

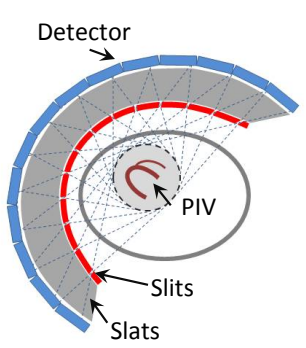
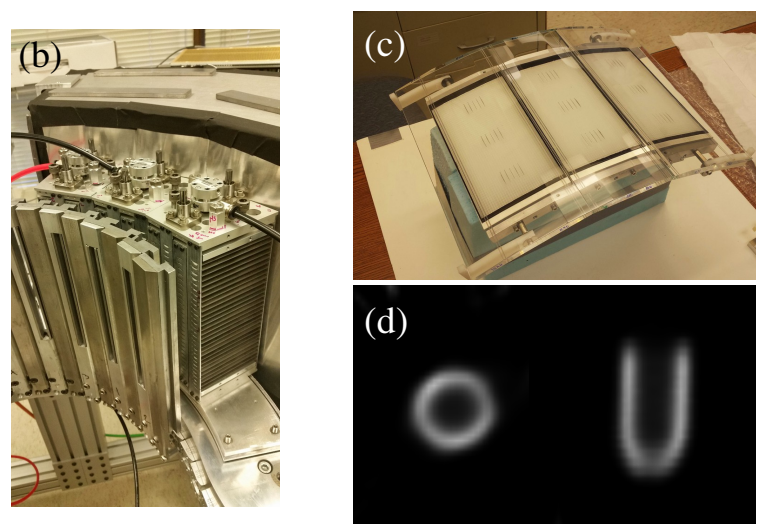

Figure 1: C-SPECT design (a), slit-slat collimator (b), detector with light guide (c), and a cardiac-insertphantom image reconstruction using a calibration system matrix (d).

dose. The higher resolution for same sensitivity can be utilized for more detailed images that could offer better or earlier diagnosis within the constraints of motion (patient, cardiac, and respiratory). The acquired stationary simultaneous projections, in combination with higher-senstivity collimation, offer dynamic-imaging functionality. A built-in low-power CT source allows for attenuation correction and quantification. The CT rays are detected by the same detection system. Other advantages of C-SPECT include low cost, smaller footprint, and more comfortable patient position. Figure 1(a) shows a schematic of C-SPECT. We present here the main components of the system.

Gantry: The design of the gantry as well as the determination of the optimal PIVs is based on real patient data from SPECT-CT studies that connect the actual thorax geometry of the patient to the relative location and size of the heart [2]. A statistical analysis of the data and the requirement that we can scan $95 \%$ of the patient population determined the shape of the gantry.

Collimator: There are several slit configurations in the current design, including a highresolution one (slit width: $\sim 3.5 \mathrm{~mm}$, total efficiency: $\sim 6 \times 10^{-4}$, resolution: $\sim 11 \mathrm{~mm}, 16$ projections) and a scout one (slit width: $\sim 6.5 \mathrm{~mm}$, total efficiency $\sim 11 \times 10^{-4}$, resolution $\sim 33 \mathrm{~mm}, 9$ projections). The latter is used for the rough location of the heart before placing it inside the PIV and it is combined with a 6-mm slat spacing. The former configuration utilizes a 3-mm slat spacing. The slat collimator is placed behind the slits and in front of the detector crystal, as shown in Figure 1(b). The resolutions and efficiencies are estimated inside the PIVs, which have diameters of $13.5 \mathrm{~mm}$ and $27 \mathrm{~mm}$ for high-resolution and scout imaging respectively. Simulation studies [3] suggest that the high-sensitivity slit collimator offers better contrast recovery, whereas the high-sensitivity slit collimators we investigated offer better lesion detectability, for a range of cardiac lesions with reduced activity compared to healthy myocardium. In addition to the above SPECT-mode collimators, an anti-scatter grid of lead blinds is used for the CT scan.

Detector: The desired curved shape of the system, in addition to the need to minimize the dead area at the edges of the detectors, led to the choice of pixelated detectors, which are made of 1-cmthick $\mathrm{NaI}(\mathrm{Tl})$ crystal scintillator [4]. The pixel pitch is $2.5 \mathrm{~mm}$ (transversely) by $3 \mathrm{~mm}$ (axially), and there are 36 (transverse) by 56 (axial) pixels. A light guide behind the detector collects and spreads the scintillated light onto a photomultiplier-tube (PMT) array (overall 130 PMTs). Figure 1(c) shows three detector modules with light guide attached.

Data Acquisition System: The PMTs are handled by 40-MHz, 12-bit ADCs as part of our OpenPET-based [5] DAQ system. It utilizes custom FPGA code to provide the necessary signal 
processing for triggering, pixel identification, and pile-up correction that is especially necessary for the higher count rates in the CT operation. The pixel position of each detected photon is determined by incorporating Anger logic, related to weighing the output from all PMTs, and with a pre-loaded decoding map. The data are organized in two-dimensional projections.

Image Reconstruction: The projections are fed into our custom-made three-dimensional iterative reconstruction with resolution recovery. A system matrix that connects the patient voxel space to the detector pixel space is constructed with calibration using an underlying analytic-model method, although MC-based system matrices have also been used. Several studies of ideal number of iterations that preserves maximal information without introducing artifacts have been performed.

\section{First Laboratory Prototype Results}

An initial laboratory prototype consisting of three modules has been constructed [6]. The prototype was mounted on a preliminary gantry and conveyer; the slit interchange system as well as the slat collapsing hydraulics were tested successfully. Its operation was a proof of concept that also provided validation and calibration data. Comparisons between MC-based expectation and experimental data validated both our system and simulation. The projections, resolution, and sensitivity of the system were as predicted by MC. Moreover, we were able to reconstruct first images using emission data and a MC-based system matrix. Figure 1(d) shows a reconstructed cardiac-insert phantom using the high-resolution collimator configuration and a calibration-based system matrix. Calibration of the system was performed with a grid of point sources, and the calibration data were used for construction of data-based analytic system matrix, which provided reconstructed images similar to the MC-based system matrix.

\section{Current Status and Conclusions}

After the successful construction of the initial laboratory prototype, we are in the process of assembling the full 14-module pre-clinical system [6]. This next stage will allow us to study the full functionality and performance of the designed system in SPECT and CT modes, including its DAQ behavior and fast real-time reconstruction capabilities. The ultimate goal is a first pre-clinical prototype and evaluation of the system performance in realistic clinical conditions.

\section{References}

[1] W. Chang, C. E. Ordonez, H. Liang, Y. Li, and J. Liu, IEEE Trans. Nucl. Sci. 56(5): 2659-71 (2009).

[2] M. Rozler, J. Halama, and W. Chang, J Nucl Med. 55:2147P (2014).

[3] J. Strologas, S. D. Metzler, Xiaofen Zheng, M. Rozler, W. Chang, IEEE NSS/MIC Conference Record (2014).

[4] M. Rozler, H. Liang, and W. Chang, IEEE Trans. Nucl. Sci. 59(5): 1831-40 (2012).

[5] W. Moses, S. Buckley, C. Vu, Q. Peng, N. Pavlov, W.S. Choong, J. Wu, C. Jackson, IEEE Trans. Nucl. Sci., 57 (5): 2532-37 (2010).

[6] W. Chang, M. Rozler, P. Sankar, D. Stentz, J. Strologas, R. Arseneau, S. Metzler, IEEE NSS/MIC Conference Record (2015). 